or even to make the notification. Oriented Dispensation - it is of paramount importance and the pharmacist's legitimate responsibility that this patient initiates his treatment with all the necessary information: the clarification on the duration of treatment, the importance of being done correctly, the transmissibility of the disease as well as the importance the treatment of the partner (s) for the success of the conduct. Pharmaceutical consultation - classify the risk of not adhering to treatment and use convincing strategies considering that any may be the reasons for resistance to treatment and qualified listening from a professional with the technical expertise to evaluate case by case and sensitivity to conduct the situation can make the difference in completing this treatment.

Results and Conclusion The analysis makes it possible to perceive how the routine of the pharmacist integrated into the Family Health offers several tools that make the conclusion of the treatment in an appropriate way of the pharmacist's responsibility.

\section{P2.42 EVALUATION OF COTRIMOXAZOLE USE AS A PREVENTIVE THERAPY AMONG PATIENTS LIVING WITH HIVIAIDS IN GONDAR UNIVERSITY REFERRAL HOSPITAL, NORTHWESTERN ETHIOPIA: A RETROSPECTIVE CROSS- SECTIONAL STUDY}

Tadesse Melaku Abegaz TM. Department of Clinical Pharmacy, School of Pharmacy, College of Medicine and Health Sciences, University of Gondar, Gondar, Ethiopia

\subsection{6/sextrans-2017-053264.218}

Introduction Cotrimoxazole preventive therapy (CPT) is a feasible, inexpensive, and well-tolerated way for patients living with HIV/AIDS to reduce HIV/AIDS-related morbidities. The aim of this study was to evaluate the use of cotrimoxazole as a prophylaxis therapy among patients living with HIV/AIDS at Gondar University Referral Hospital (GURH), northwestern Ethiopia.

Methods A retrospective cross-sectional study was conducted at GURH, from September 2013 to October 2015. Medical records of 264 patients were selected by using systematic random sampling technique. Data were collected using the structured checklist and evaluated against World Health Organisation (WHO) guidelines. The quantitative data were analysed using the statistical packages for social sciences Version 20. Descriptive and binary logistic regressio were used to assess the association between different variables.

Results Approximately 95 (36.0\%) patients were at WHO clinical stage III at the start of CPT. The use of CPT was consistent with the guidelines in the rationale for indication 200 (75.75\%) and dose 263 (99.62\%), despite the presence of contraindications in $24(9.90 \%)$ patients. The occurrence of cotrimoxazole-associated side effects was higher in the first month of therapy.

Conclusion Although the practice of discontinuation of CPT and follow-up for adverse drug effects were not consistent with WHO guidelines on the rational use of cotrimoxazole prophylaxis, the use of CPT among people living with HIV/ AIDS at GURH was appropriate. Health professionals should adhere to the available updated guidelines to reduce the occurrence of adverse effects.
P2.43 GENDER DIFFERENCES IN RISK FACTORS AND CLINICAL OUTCOMES OF PATIENTS RECEIVING ANTIRETROVIRAL THERAPY AT AN HIV CLINIC IN GUATEMALA CITY OVER A 9-YEAR PERIOD

Theresa Tharakan, Matt Anderson. Albert Einstein College of Medicine, Bronx, USA

\subsection{6/sextrans-2017-053264.219}

Introduction There is no consensus on gender differences in clinical outcomes of HIV-infected patients. Immunologic, virologic, and survival data for patients receiving antiretroviral therapy (ART) show an inconsistent presence and direction of a gender gap. Gender and sexual behavior-based outcomes analysis is lacking in Guatemala, which has largely sexual transmission of HIV. We examine outcomes of HIV-positive Guatemalans receiving ART over a 9 year period.

Methods Retrospective cohort analysis was conducted using a database of treatment-naive patients offered free ART at the Clinica Familiar Luis Angel Garcia in Guatemala City from 2004 to 2014. Multivariate Cox regression was used to study gender differences in all-cause mortality, immunologic failure (CD4 $<100$ cells $/ \mu \mathrm{L}$ twice or CD4 < baseline) and virologic suppression (viral load $<50$ HIV-1 RNA copies/mL within 1 year of starting ART).

Results 4248 patients were included: 2605 men, 1617 women, and 26 transgender patients (analysed separately). Compared to men, women had higher median CD4 counts (198 vs. 126 cells $/ \mu \mathrm{L}, \mathrm{p}<0.001)$ and lower median viral loads $(6.48 \times$ $10^{4}$ copies $/ \mathrm{mL}$ vs. $11.27 \times 10^{4}$ copies $\left./ \mathrm{mL}, \mathrm{p}<0.001\right)$ at baseline. In multivariate analysis, mortality decreased with female gender (HR 0.52, 95\% CI 0.29-0.93, p=0.029) while it increased with age (HR 1.02, 95\% CI 1.003-1.04, $\mathrm{p}=0.02$ ) and inconsistent condom use (HR 9.36, 95\% CI 2.61-33.63, $\mathrm{p}=0.001)$. In women alone, these factors did not predict mortality. In men alone, mortality increased with inconsistent condom use (HR 23.26, 95\% CI 2.89-187.3, p=0.003), and number of sexual partners (HR 1.02, 95\% CI 1.001-1.039, $\mathrm{p}=0.041)$. Gender did not predict immunologic failure. Female gender predicted a lower rate of viral suppression (HR $0.6,95 \%$ CI $0.41-0.85, \mathrm{p}=0.005$ ).

Conclusion Women receiving ART have lower mortality than men when adjusted for sociodemographic factors and sexual behaviours. Sexual risk factors affect genders differently and can predict treatment outcomes in previously infected patients.

\section{\begin{tabular}{|l|l}
\hline P2.44 NO DIFFERENCES IN KNOWLEDGE OF KEY HIV TEST \\
\hline
\end{tabular} CONCEPTS BETWEEN USERS OF AN ONLINE STI TESTING SERVICE (GETCHECKEDONLINE.COM) AND IN- CLINIC TESTERS IN VANCOUVER, CANADA}

${ }^{1}$ Travis Salway, ${ }^{1}$ Kimberly Thomson, ${ }^{2}$ Darlene Taylor, ${ }^{3}$ Elizabeth Elliot, ${ }^{4}$ Tom Wong, ${ }^{5}$ Christopher Fairley, ${ }^{1}$ Devon Haag, ${ }^{1}$ Troy Grennan, ${ }^{6}$ Jean Shoveller, ${ }^{1}$ Gina Ogilvie, ${ }^{1}$ Mark Gilbert. ${ }^{1}$ British Columbia Centre for Disease Control, Vancouver, Canada; ${ }^{2}$ University of British Columbia Okanagan, Kelowna, Canada; ${ }^{3}$ College of Registered Nurses of British Columbia, Vancouver, Canada; ${ }^{4}$ Health Canada, Ottawa, Canada; ${ }^{5}$ University of Melbourne, Melbourne, Australia; ${ }^{6}$ University of British Columbia, Vancouver, Canada

\subsection{6/sextrans-2017-053264.220}

Introduction Online HIV/STI testing is an alternative to inclinic testing, but may lead to missed opportunities for education due to the lack of provider-delivered pre/post-test counselling. GetCheckedOnline (GCO) is an online testing service offered through an urban STI clinic in Vancouver. It was 